\title{
Craniofacial growth and respiration: a study on an animal model
}

\author{
Luca Levrini, MD,DDSc,MSc ${ }^{1}$ \\ Alessandro Mangano, DDS,MSc ${ }^{1}$ \\ Alessandro Ambrosoli, DDS, MSc ${ }^{1}$ \\ Paola Merlo, DDS, MSc ${ }^{1}$ \\ Carlo Mangano, MD,DDSc ${ }^{2}$ \\ Alberto Caprioglio, DDS,MSc ${ }^{1}$ \\ 1 Department of Surgical and Morphological Sci- \\ ences, University of Insubria, Varese, Italy \\ 2 Private practice
}

Corresponding author:

Alessandro Mangano

Department of Surgical and Morphological Sciences, University of Insubria

Via Ravasi, 2

21100 Varese, Italy

E-mail: ale.mangano10@gmail.com

\section{Summary}

Aims. The aim of this study was to analyse the effects of nasal obliteration with regards to the linear dimensions of dissected hemimandibles of a homogeneous sample of young rats.

Methods. 68 pure breed male Sprague-Dawley rats, aged four weeks, were divided into four groups of 17: two control groups and two test groups. The first control group was sacrificed at the beginning of the observation period and the other one at the end. The test groups, one of which had the right nostril occluded by silicon material while the other had the left occluded, were sacrificed after eight weeks, at twelve weeks. After isolating the hemi-mandibles, four vertical and four sagittal measurements were taken; comparison was then made between the control groups and the experimental groups. The sagittal measurements are articular surface of the condyle-neck incisor (SARCIN), articular surface of the condyle-mental foramen (SARFORO), articular surface of the condyle-margo incisalis (SARMI), articular surface of the condyle-surface mesial of the first molar (SARSMM). The vertical measurements are superior condyle surface-base (SCOSUB), mesial surface of the first molar-base (SUMESM), maximum inferior arched concavity-base, (XCOARIB), maximum sigmoid notch concavity-base (XCOINSB).

Results. The sagittal and vertical measurements showed an increase in the values of the experimental group when compared to the controls.
Conclusion. An altered nasal respiration is able to influence the patterns of facial growth and in particular to induce an increase in the growth of the mandible.

Key words: craniofacial growth, respiration, orthodontics, growth patterns.

\section{Introduction}

Numerous studies conducted on animals have demonstrated a strict correlation between craniofacial morphogenesis and the onset of functional anomalies (1). It has been shown that in bipedal rats (created by amputation of two paws) variations in body and head posture have been brought about, with consequential alterations in the craniofacial region (2). These variations consist above all in an opening of the angle of the base of the skull and an anterior displacement of the occipital foramen. A theory has been put forward that the craniofacial alterations may occur as a consequence of a lack in nasal airflow, which is independent from the onset of oral respiration (3-5). Significant correlation between oral respiration and craniofacial modifications were found in a study carried out on a sample of 80 rats, in which one or both nostrils were occluded. Besides a reduction in overall growth of the animals, where a clear-cut ponderal reduction was shown, a reduction in the general dimensions of the skull was also demonstrated. Important variations were found not only in the mandible but also in the pterygoid and tympanal areas. In particular, a postrotation of the viscerocranium and of the mandible were noted, probably due to induced oral respiration. In fact, under these conditions, the rodent is forced to an obligatory raising of the head in order to increase the opening of the pharynx (6). In another study made on rats, where a monolateral nostril occlusion had been carried out, a significant deviation of the midline towards the side of the occlusion was noticed. This has therefore led to the belief that a lack of airflow in the occluded fossa inhibits morphogenesis and, therefore, the passage of airflow through the nasal cavity constitutes in itself a morphogenetic stimulus for the viscerocranium development (7).

The anatomic and structural differences between man and other animals often make it difficult to compare the results obtained in these studies $(8,9)$, particularly when comparing human anatomy with rodents' anatomy. From this point of view, studies made on primates would appear to be much more reliable $(10,11)$. In all mammals respiration requires a 
highly specialised structure able to adapt to the variety of needs of every specie and to the different evolutionary pressures (12). In the albino rat, even though skeletal segments are comparable in number to those of the human skull (13), the respiratory function is carried out differently. The topographic proximity between the epiglottis and the soft palate makes the passage of air through the oral cavity extremely difficult thus making the rat's respiration mainly nasal. Many studies conducted on human patients (14-28) have shown cephalometrically a correlation between respiration and craniofacial development, but also an interesting possible correlation between growth hormone levels and the morphologic development of the upper and lower jaw (15).

A very recent study conducted on newborn rats (29) showed that a diet-induced hypermethioninemia in rat dams results in growth retardations and histomorphological changes of the spheno-occipital synchondrosis, an important craniofacial growth centre in newborns: this may elucidate facial dysmorphoses reported in patients suffering from hypermethioninemia.

The hypothesis for this study is the possibility that respiratory function may also modify skeletal growth in the animal model. The type of modification induced is not clinically relevant, due to the reasons cited above concerning the lack of comparison possibilities between man and rat.

\section{Materials and methods}

This study was carried out according to the declaration of Helsinki (1964). In this study 68 male pure breed Sprague-Dawley rats were used. At the beginning of the experiment the rats were 4 weeks old. The animals have been randomly divided into four groups of 17 (two experimental and two controls); of the two experimental group, one had the right nostril occluded with silicone material, while the other had the left nostril occluded. The animals belonging to two control groups (groups C and D) had no nostril occluded and were sacrificed at two different times; respectively after 4 weeks and after 12 weeks. Accommodation was made putting five rats per cage with water and food at their disposal (Tab. 1). A Student t-test was applied. The level of significance was set at 0.05. All statistical analyses were run on the statistical package SPSS (SPSS 17.0; SPSS Inc., Chicago, IL,USA).

At the beginning of the observation period rats belonging to Group C were sacrificed, in order to verify whether the growth of the rats was homogeneous or not: the measurements were quite the same, with a t- test $p=0.03$. Using a general anaesthesia for animals (Chetamine cloridate i.m.) the nostrils of groups A and B were occluded with silicon material. In order to verify a comparison between the experimental models and evaluate whether different ways of breathing had any influence on the health of the rats they were weighed at the beginning and end of the observation period. The animals were then sacrificed at 12 weeks. Soft tissues were then carefully removed from the head until the cranium was completely isolated and the mandibles could be separated. The mandibles were then divided into two hemimandibles. Checking whether the nasal cavities had indeed been obstructed, only 29 obstructions resulted out of 31 (the total number of possibly obstructed nostrils corresponds to 31 due to the fact that 3 out of the 34 rats with obstructed nostrils had died). The final number of specimens of mandible was 29 because two rats' nasal cavities were not totally obstructed and were discarded from the study. The 29 useful hemi-mandibles were photographed using a digital camera (Polaroid Digital Microscope Camera): they were put onto the stage plate of a stereomicroscope, on a black background with a millimetre marker and identity signs for the groups (Fig. 1); in order to guarantee the same position of the hemimandibles during the analysis, the latter where glued to a support that made the external face of the mandible parallel to the surface of the stage plate. The photographs were then transferred to a software system (IMAT R-D) which allowed measurements up to approximately $0.1 \mathrm{~mm}$ to be taken. The linear measurements were divided into sagittal (S) and vertical (V); we decided not to evaluate the thickness of the mandibles because we considered that this value was not useful to the purpose of our research, in that

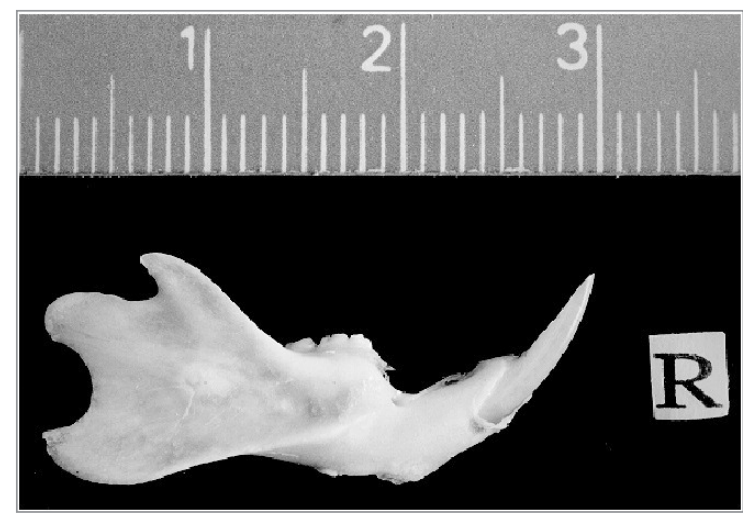

Figure 1. One of the sample analyzed.

Table 1. Subdivision of the rats in the four groups.

\begin{tabular}{llllll}
\hline & Group type & Number of rats & Nostril occluded & Sacrifice time & Average weight \\
\hline Group A & experimental & 17 & left & 12 weeks & Gr $384 \pm 23$ \\
Group B & experimental & 17 & right & 12 weeks & Gr $395 \pm 32$ \\
Group C & control & 17 & none & 4 weeks & Gr $72 \pm 19$ \\
Group D & control & 17 & none & 12 weeks & Gr $388 \pm 27$ \\
\hline
\end{tabular}


the volume of the superior aerial column shall not be modified by the thickness of this particular bone.

The acquired data concerning the linear measurements measured on the rats' mandible may be divided into two main groups: sagittal measurements (Fig. 2) and vertical measurements (Fig. 3).

With regard to the vertical values they were identified perpendicular to a reference line passing from the upper reference point, the following distances were considered:

V1: Superior condyle surface - Base (SCOSUB)

V2: Maximum concavity sigmoid notch - Base (XCOINSB)

V3: Medial surface of the first molar - Base (SUMESM)

V4: Maximum concavity inferior arch - Base (XCOARIB)

With regard to the sagittal values, the following distances were considered (Figure below):

S1: Articular surface of the condyle - Incisal margin (SARMI)

S2: Articular surface of the condyle - Medial surface of the first molar (SARSMM)

S3: Articular surface of the condyle - Incisal neck (SARCIN)

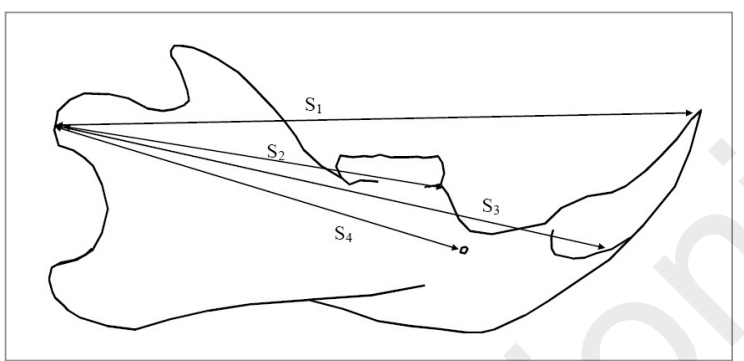

Figure 2. Sagittal measurements.

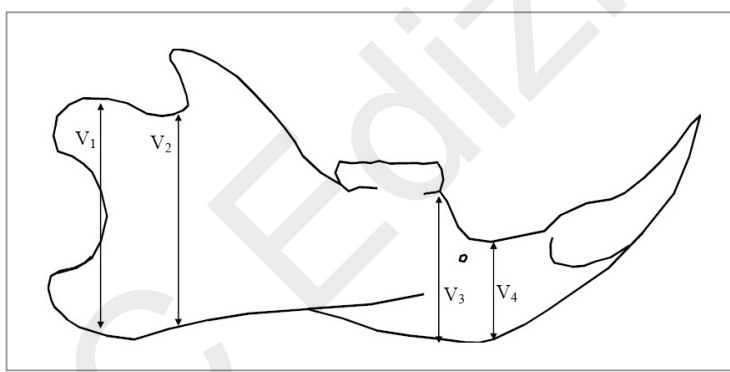

Figure 3. Vertical measurements.
S4: Articular surface of the condyle - Mental foramen (SARFORO)

The average, standard deviation, mode and median were calculated for every variable measured. The statistical significance of the increases in evidence were ascertained with the use of t-test $(p<0.05)$ for correlated samples (matched data) in the sample and the control group. Due to the uniform genetic and phenotypical characteristics of the sample it was not necessary to apply non parametric statistical tests, where the conclusions obtained are valid independently from the normality of the population and the homogeneity of the variance between the groups. The application of the t-test was, therefore, considered to be sufficient.

\section{Results}

The sagittal measurements demonstrate a homogenous increase in the values together with a reduction of the standard deviations $(p<0.05)$. The SARCIN values increase compared to the control; after 8 weeks of obstruction in the upper nasal airways, a noticeable increase in the values and a decrease of the standard deviation was observed.

The values of SARFORO, SARMI and SARSMM also present a similar trend with an increase in the distances and a compression of the standard deviations (Tab. 2).

When analysing the data concerning the vertical distances a tendency towards an increase in the linear values is noted, accompanied by a modest growth in the standard deviation.

The values SUMESM, XCOARIB, and XCOINSB all demonstrate an increase in the linear dimensions and a modest increase in the standard deviation.

Three rats died after nasal obstruction and, as already said before, the real obstruction of the nasal cavities was found in 29 out of 31 . Considering the weight, no significant differences were observed between the test group and the control group.

\section{Discussion}

The lack of experimental studies on the modification of maxillofacial growth in rats following obstruction of

Table 2. Measurements found analysing the rats after they were sacrificed.

\begin{tabular}{|c|c|c|c|c|c|c|}
\hline & \multicolumn{3}{|c|}{ Control } & \multicolumn{3}{|c|}{ Experimental } \\
\hline & Mean $(\mathrm{mm})$ & S.D. & T-Test $p<0.05$ & Mean (mm) & S.D. & T-Test $\mathrm{p}<0.05$ \\
\hline SARCIN & 24,5 & 0,6100 & 0 & 24,8 & 0,6080 & 0 \\
\hline SARFORO & 20,2 & 0,6661 & 0 & 20,5 & 0,4740 & 0 \\
\hline SARMI & 29,9 & 0,5690 & 0 & 29,9 & 0,8480 & 0 \\
\hline SARSMM & 18,4 & 0,4930 & 0 & 18,7 & 0,3760 & 0 \\
\hline ScosuB & 11,5 & 0,4410 & ns & 11,5 & 0,4500 & ns \\
\hline SUMESM & 6,8 & 0,2150 & 0 & 7 & 0,3930 & 0 \\
\hline XCOARIB & 4,6 & 0,1586 & 0 & 4,8 & 0,2650 & 0 \\
\hline XCOINSB & 10,7 & 0,3750 & 0 & 10,9 & 0,4080 & 0 \\
\hline
\end{tabular}


the primary nasal airways does not allow us to correlate our collected data with pre-existing data except for the publications of Deli and colleagues of 1991 and 1992 (2, 3). However there are many articles in the literature investigating the craniofacial modifications occurring in humans with breathing alterations during the growing phase (18-22). Zucconi et al. (18) showed how patients with high values of mandibular angle had a posterior rotation of the mandible resulting in minor craniofacial anomalies. The role of nasorespiratory characteristics, it's influence on respiratory patterns and the diagnostic tools used to investigate this condition have been debated for years (2325). Kluemper et al. indicated that cephalometric evaluation can be considered a poor diagnostic tool when it comes to objectively evaluate a nasal impairment (23). Many investigators showed how the respiratory function is capable of influencing the growth of craniofacial structures and how a respiratory impairment has an important effect on the development and growth of these structures (25-28). A correlation between the growth pattern and respiration has been established $(18,26,27)$ showing how a retropositioned mandible induces a vertical growth of the face resulting in a final incremented vertical dimension $(18,26,27)$.

In our study the data showed how the respiratory pattern was able to affect the growth of the facial structures. In the rat, a decrease or in any case a negative alteration of the opening of the superior airways significantly modifies the patterns of structural growth of the mandible, although we are speaking of differences in the range of tenths of millimetres (30).

Moreover, the sagittal values, with a reduction of their standard deviations, show that anomalies in respiratory function induce an increase in mandible growth and also that the reduction of air flow is able to level out the test group almost reducing the individual variability of growth of physiologically developed rats.

The animals demonstrate a growth behaviour more similar in the pathology rather than in the complete anatomic structures.

Although it is extremely complex to identify the pathogenic mechanisms that cause growth alteration in correlation to reduced nasal respiration, it would seem possible to assume that an important factor for the increased sagittal development of the mandible could be found in the lowering of lingual posture. Analogically the increase in skeletal verticality would appear to confirm data referring to more complex mammals with post-rotation induced by the new postural order of the skull and the mandible. An altered nasal respiration is able to influence the patterns of facial growth and in particular to induce an increase in the growth of the mandible in rats.

From a clinical point of view, confirmation of the anatomical-functional correlation of the animal model of the rat, much more basic compared to the complexity of primates, further emphasizes the need for other investigations, mainly on the human being, about the correlations between respiratory functions and skeletal development of the splanchnocranium.

\section{References}

1. Babero BB, Yousef MK, Wawerna JC. Comparative histology of the respiratory apparatus of three desert rodents and the albino rat: a view on morphological adaptations. Comp Biochem Physiol A. 1973;44:585-597.

2. Deli R, Deli G, Germano D, Asciutto T. Craniofacial growth after experimental nasal obstruction in rats. J Dent Res. 1991;760:70.

3. Deli R, Deli G, Ursini R. Nasal obstruction and cranio-facial growth. Eur J Orthod. 1992;14:76.

4. Harvold EP, Vargervik K, Chierici G. Primate experiments on oral respiration. Am J Orthod. 1981;79:359-73.

5. Nespoli L, Caprioglio A, Brunetti L, Nosetti L. Obstructive sleep apnea syndrome in childhood. Early Hum Dev. 2013;3:33-37.

6. Moss ML. Rotation of the otic capsule in bipedal rats. Am J Phys Anthropol. 1961:19:301-7.

7. Schlenker WL, Jennings BD, Jeiroudi MT, Caruso JM. The effects of chronic absence of active nasal respiration on the growth of the skull: a pilot study. Am J Orthod Dentofacial Orthop. 2000;117:15-27.

8. Song HG, Pae EK. Changes in orofacial muscle activity in response to changes in respiratory resistance. Am J Orthod Dentofacial Orthop. 2000;119:22-32.

9. Svendsen T, Hau J. Handbook of laboratory animals science. Florida: CRC Press Inc. 1994.

10. Tomer BS, Harvol EG. Primate experiments on mandibular growth direction. Am J Orthod. 1982;82:114-9.

11. Vidić B, Taylor JJ, Rana MW, Bhagat BD. The respiratory glandular system in the rat's lateral nasal wall in normal and polluted environments. Verh Anat Ges. 1971;66:83-85.

12. Weijs, WA. Mandibular movements of the albino rat during feeding. J Morphol. 1975;145:107-124.

13. Youseff, EH. The chondrocranium of the albino rat. Acta Anatomica. 1966;64:586-617.

14. Bakor SF, Enlow DH, Pontes P, De Biase NG.. Craniofacial growth variations in nasal-breathing, oral-breathing, and tracheotomized children. Am J Orthod Dentofacial Orthop. 2011 Oct; 140(4):486-92.

15. Peltomäki T. The effect of mode of breathing on craniofacial growth revisited. Eur J Orthod. 2007 Oct; 29(5):426-9.

16. Bakor SF, Enlow DH, Pontes P, De Biase NG.. Craniofacial growth variations in nasal-breathing, oral-breathing, and tracheotomized children. Am J Orthod Dentofacial Orthop. 2011 Oct;140(4):486-92.

17. Harari D, Redlich M, Miri S, Hamud T, Gross M. The effect of mouth breathing versus nasal breathing on dentofacial and craniofacial development in orthodontic patients. Laryngoscope. 2010 Oct;120(10):2089-93.

18. Zucconi M, Caprioglio A, Calori G, Ferini-Strambi L, Oldani A, Castronovo C, Smirne S. Craniofacial modifications in children with habitual snoring and obstructive sleep apnoea: a case-control study. Eur Resp J. 1999;13(2):411-417.

19. D’Ascanio L, Lancione C, Pompa G, Rebuffini E, Mansi N, Manzini M. Craniofacial growth in children with nasal septum deviation: a cephalometric comparative study. Int J Pediatr Otorhinolaryngol. 2010 Oct;74(10):1180-3. Epub 2010 Aug 9.

20. Sousa JB, Anselmo-Lima WT, Valera FC, Gallego AJ, Matsumoto MA. Cephalometric assessment of the mandibular growth pattern in mouth-breathing children. Int $\mathrm{J}$ Pediatr Otorhinolaryngol. 2005 Mar;69(3):311-7. Epub 2004 Dec 15.

21. Mattar SE, Anselmo-Lima WT, Valera FC, Matsumoto MA. Skeletal and occlusal characteristics in mouth-breathing pre-school children. J Clin Pediatr Dent. 2004 Summer;28(4):315-8.

22. Kawashima S, Peltomäki T, Sakata H, Mori K, Happonen RP, Rönning O. Craniofacial morphology in preschool children with sleep-related breathing disorder and hypertrophy of tonsils. Acta Paediatr. 2002;91(1):71-7. 
23. Kluemper GT, Vig PS, Vig KW. Nasorespiratory characteristics and craniofacial morphology. Eur J Orthod. 1995 Dec;17(6):491-5.

24. Principato JJ. Upper airway obstruction and craniofacial morphology. Otolaryngol Head Neck Surg. 1991 Jun;104(6):88190. Review.

25. Klein JC. Nasal respiratory function and craniofacial growth Arch Otolaryngol Head Neck Surg. 1986 Aug;112(8):843-9.

26. McNamara JA. Influence of respiratory pattern on craniofacial growth. Angle Orthod. 1981 Oct;51(4):269-300.

27. Woodside DG, Linder-Aronson S, Lundstrom A, McWilliam J. Mandibular and maxillary growth after changed mode of breath- ing. Am J Orthod Dentofacial Orthop. $1991 \mathrm{Jul} ; 100(1): 1-18$

28. Jennings BD, Schlenker WL, Boyne PJ, Walters RD. The effects of chronic absence of active nasal respiration on the growth of the skull. Prog Clin Biol Res. 1985;187:403-12. No abstract available.

29. Linder-Aronson S, Woodside DG, Lundström A. Mandibular growth direction following adenoidectomy. Am J Orthod. 1986 Apr;89(4):273-84.

30. Römer $P$, Weingärtner J, Desaga $B$, Kubein-Meesenburg $D$ Reicheneder C, Proff P. Effect of excessive methionine on the development of the cranial growth plate in newborn rats. Arch Oral Biol. 2012 Sep;57(9):1225-30. 